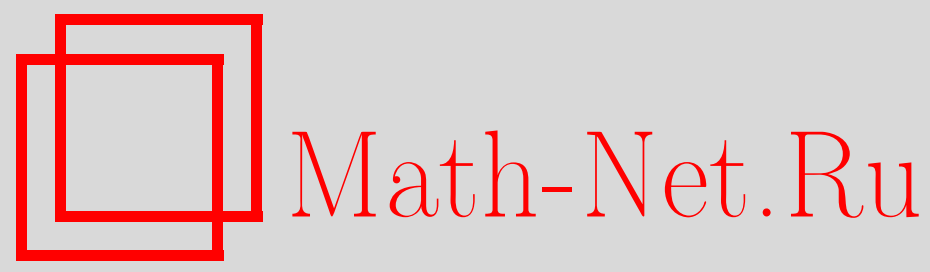

В. Е. Федоров, О. А. Рузакова, Одномерная и двумерная управляемость уравнений соболевского типа в банаховых пространствах, Матем. заметки, 2003, том 74, выпуск 4, 618-628

DOI: https://doi.org/10.4213/mzm283

Использование Общероссийского математического портала Math-Net.Ru подразумевает, что вы прочитали и согласны с пользовательским соглашением http://www.mathnet.ru/rus/agreement

Параметры загрузки:

IP: 3.81 .55 .215

26 апреля 2023 г., $07: 31: 20$

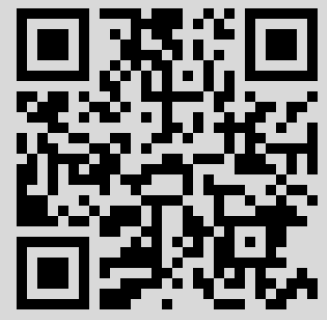




\title{
ОДНОМЕРНАЯ И ДВУМЕРНАЯ УПРАВЛЯЕМОСТЬ УРАВНЕНИЙ СОБОЛЕВСКОГО ТИПА В БАНАХОВЫХ ПРОСТРАНСТВАХ
}

В.Е. Федоров, О. А. Рузакова

\begin{abstract}
Для сингулярных линейных дифференциальных уравнений первого порядка в банаховых пространствах получены необходимые условия одномерной $\varepsilon$-управляемости. Это является обобщением аналогичных результатов для регулярных уравнений. Показано, что для рассматриваемого класса уравнений более естественным является понятие двумерной $\varepsilon$-управляемости, для которой аналогичные необходимые условия являются также и достаточными. С помощью абстрактных результатов получены достаточные условия двумерной $\varepsilon$-управляемости задачи Коши-Дирихле для уравнения Баренблатта-Желтова-Кочиной.

Библиограффия: 9 названий.
\end{abstract}

1. Введение. Пусть $\mathscr{X}, \mathscr{Y}$ - банаховы пространства, оператор $L \in \mathscr{L}(\mathscr{X} ; \mathscr{Y})$ (т.е. линейньй непрерьвньй), $\operatorname{ker} L \neq\{0\}$, оператор $M \in \mathscr{C l}(\mathscr{X} ; \mathscr{Y})$ (линейньй замкнутьй, плотно определенньй в $\mathscr{X})$, функция $u(\cdot):[0, T] \rightarrow \mathbb{R}$, вектор $b \in \mathscr{Y}$. Рассмотрим задачу Коши $x(0)=x_{0}$ для уравнения соболевского типа

$$
L \dot{x}(t)=M x(t)+b u(t), \quad 0 \leqslant t \leqslant T .
$$

Она представляет собой абстрактную форму многих задач, в том числе начально-краевых задач для уравнений и систем уравнений математической физики с вырожденным оператором при старшей производной по времени [1], [2]. Систематически изучать такие задачи первьм начал Соболев [3] (см. также [4]), поэтому уравнения вида (1) часто назьвают уравнениями соболевского типа. Обоснование использования такой терминологии см. в [5].

В теории дифференциальных уравнений очень важньм является понятие управляемости уравнения [6], т.е. приведения траектории его решения в наперед заданную точку. В случае банаховых пространств речь может идти только о приведении траектории в $\varepsilon$-окрестность заданной точки. В работе [7] рассмотрено уравнение в банаховом пространстве

$$
\frac{d x}{d t}=A x+b u
$$

с линейным ограниченным оператором $A$, вектором $b \in \mathscr{X}$ и функцией $u:[0, T] \rightarrow \mathbb{R}$, a также вопросы $\varepsilon$-управляемости для систем, описьваемых такими уравнениями, т.е. так

Работа частично поддержана Государственной научной стипендией для молодых ученых. 
назьваемой одномерной $\varepsilon$-управляемости (поскольку функция управления действует в одномерное пространство). Цель данной работы - на основе имеющихся результатов об $\varepsilon$-управляемости уравнения (2) исследовать одномерную $\varepsilon$-управляемость уравнения (1) с $(L, \sigma)$-ограниченным оператором $M[1]$.

В данной работе показано, что для вырожденного уравнения (1) понятие $\varepsilon$-управляемости в нуль отличается от понятий $\varepsilon$-управляемости из нуля и $\varepsilon$-управляемости из любой точки в любую. В то же время последние два понятия эквивалентны. Кроме того, получены необходимые условия одномерной $\varepsilon$-управляемости уравнения (1).

Замечено, что для уравнений соболевского типа более естественно рассматривать задачу двумерной $\varepsilon$-управляемости, т.е. задачу $\varepsilon$-управляемости уравнения

$$
L \dot{x}(t)=M x(t)+b^{0} u_{0}(t)+b^{1} u_{1}(t), \quad 0 \leqslant t \leqslant T .
$$

В работе получены необходимые и достаточные условия двумерной $\varepsilon$-управляемости этого уравнения.

В качестве примера рассмотрена задача Коши-Дирихле для уравнения Баренблатта-Желтова-Кочиной, для которой получены достаточные условия двумерной $\varepsilon-$ пправляемости.

2. Относительно спектрально ограниченный оператор. Приведем необходимые для дальнейшего изложения вспомогательные результаты, доказательства которых можно найти в [1].

Пусть $\mathscr{X}, \mathscr{Y}$ - банаховы пространства. Через $\mathscr{L}(\mathscr{X} ; \mathscr{Y})$ будем обозначать банахово пространство линейньх непрерьвных операторов $A: \mathscr{X} \rightarrow \mathscr{Y}$. Если $\mathscr{Y}=\mathscr{X}$, то обозначение сократится до $\mathscr{L}(\mathscr{X})$.

Множество линейных замкнутых операторов $A: \operatorname{dom} A \rightarrow \mathscr{Y}$ с областями определения, плотными в пространстве $\mathscr{X}$, будем обозначать $\mathscr{C l}(\mathscr{X} ; \mathscr{Y})$. Множество операторов $\mathscr{C l}(\mathscr{X} ; \mathscr{X})$ обозначим через $\mathscr{C l}(\mathscr{X})$.

В дальнейшем предполагаем $L \in \mathscr{L}(\mathscr{X} ; \mathscr{Y}), M \in \mathscr{C l}(\mathscr{X} ; \mathscr{Y})$. Обозначим

$$
\rho^{L}(M)=\left\{\mu \in \mathbb{C}:(\mu L-M)^{-1} \in \mathscr{L}(\mathscr{Y} ; \mathscr{X})\right\} .
$$

ОПРЕДЕлЕниЕ 1 . Оператор $M$ назьвается $(L, \sigma)$-ограниченным, если

$$
\exists a>0 \quad \forall \mu \in \mathbb{C} \quad(|\mu|>a) \Rightarrow\left(\mu \in \rho^{L}(M)\right) .
$$

Возьмем $(L, \sigma)$-ограниченньй оператор $M$, выберем в комплексной плоскости $\mathbb{C}$ замкнутьй контур $\gamma=\{\mu \in \mathbb{C}:|\mu|=R>a\}$. Тогда имеют смысл следующие интегралы:

$$
P=\frac{1}{2 \pi i} \int_{\gamma}(\mu L-M)^{-1} L d \mu, \quad Q=\frac{1}{2 \pi i} \int_{\gamma} L(\mu L-M)^{-1} d \mu .
$$

Операторы $P$ и $Q$ являются проекторами. Положим $\mathscr{X}^{0}=\operatorname{ker} P, \mathscr{Y}^{0}=\operatorname{ker} Q$, $\mathscr{X}^{1}=\operatorname{im} P, \mathscr{Y}^{1}=\operatorname{im} Q$. Имеем

$$
\mathscr{X}=\mathscr{X}^{0} \oplus \mathscr{X}^{1}, \quad \mathscr{Y}=\mathscr{Y}^{0} \oplus \mathscr{Y}^{1} .
$$

Через $L_{k}$ (соответственно $M_{k}$ ) обозначим сужение оператора $L$ (соответственно $M$ ) на $\mathscr{X}^{k}\left(\operatorname{dom} M_{k}=\operatorname{dom} M \cap \mathscr{X}^{k}\right), k=0,1$. Кроме того, через $\sigma_{k}^{L}(M)$ будем обозначать множество $\mathbb{C} \backslash \rho^{L_{k}}\left(M_{k}\right)-L_{k}$-спектр оператора $M_{k}$. 
Теорема 1. Пусть оператор $M(L, \sigma)$-ограничен. Тогда действия операторов

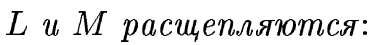

(i) $L_{k} \in \mathscr{L}\left(\mathscr{X}^{k} ; \mathscr{Y}^{k}\right), k=0,1$;

(ii) $M_{0} \in \mathscr{C l}\left(\mathscr{X}^{0} ; \mathscr{Y}^{0}\right), M_{1} \in \mathscr{L}\left(\mathscr{X}^{1} ; \mathscr{Y}^{1}\right)$;

(iii) существует оператор $L_{1}^{-1} \in \mathscr{L}\left(\mathscr{Y}^{1} ; \mathscr{X}^{1}\right)$;

(iv) $\sigma_{0}^{L}(M)=\varnothing$;

(v) cyществует оператор $M_{0}^{-1} \in \mathscr{L}\left(\mathscr{Y}^{0} ; \mathscr{X}^{0}\right)$;

(vi) существует аналитическая разрешающая әруппа $\left\{U^{t} \in \mathscr{L}(\mathscr{U}): t \in \mathbb{R}\right\}$ уравнения (1), причем $U^{t}=e^{t L_{1}^{-1} M_{1}} P$.

Введем обозначения

$$
H=M_{0}^{-1} L_{0} \in \mathscr{L}\left(\mathscr{X}^{0}\right), \quad S_{1}=L_{1}^{-1} M_{1} \in \mathscr{L}\left(\mathscr{X}^{1}\right) .
$$

Для операторнозначной функции $(\mu L-M)^{-1}$ бесконечно удаленная точка является

(i) устранимой особой точкой, если $H=\mathbb{O}$;

(ii) полюсом порядка $p \in \mathbb{N}$, если $H^{p} \neq \mathbb{O}$, а $H^{p+1}=\mathbb{O}$;

(iii) существенно особой точкой, если $\forall p \in \mathbb{N} H^{p} \neq \mathbb{O}$.

Через $\mathbb{O}$ здесь обозначен нулевой оператор, определенньй на подпространстве $\mathscr{X}^{0}$.

Бесконечность будем назьвать несущественной особой точкой порядка $p \in\{0\} \cup \mathbb{N}$, если она является устранимой особой точкой $(p=0)$ или полюсом порядка $p \in \mathbb{N}$.

ТЕОрема 2. Пусть оператор $M(L, \sigma)$-ограничен, а бесконечность - несущественная особая точка порядка р оператор-функиии $(\mu L-M)^{-1}$. Тогда для любой функиии $f(t) \in C^{p+1}([0 ; T] ; \mathscr{Y})$ и для любого начального значения

$$
x_{0} \in \mathscr{P}_{f}=\left\{x \in \mathscr{X}:(I-P) x=-\sum_{k=0}^{p} H^{k} M_{0}^{-1}(I-Q) f^{(k)}(0)\right\}
$$

существует единственное решение $x(t) \in C^{1}([0, T] ; \mathscr{X})$ задачи Коши $x(0)=x_{0}$ для уравнения $L \dot{x}(t)=M x(t)+f(t)$, имеющее вид

$$
x(t)=U^{t} x_{0}+\int_{0}^{t} U^{t-s} L_{1}^{-1} Q f(s) d s-\sum_{k=0}^{p} H^{k} M_{0}^{-1}(I-Q) f^{(k)}(t) .
$$

В основе доказательства теоремы 2 лежит используемая нами в дальнейшем редукция рассматриваемого уравнения к системе двух уравнений на подпространствах $\mathscr{X}^{1}$ и $\mathscr{X}^{0}$ соответственно, которые в случае функции $f(t)=b u(t)$ имеют вид

$$
\dot{x}^{1}(t)=S_{1} x^{1}(t)+u(t) L_{1}^{-1} b^{1}
$$

на пространстве $\mathscr{X}^{1}$,

$$
H \dot{x}^{0}(t)=x^{0}(t)+u(t) M_{0}^{-1} b^{0}
$$

на пространстве $\mathscr{X}^{0}$, где $b^{1}=Q b, b^{0}=(I-Q) b$. Действительно, уравнение (3) получается при действии на обе части уравнения (1) сначала проектором $Q$, а затем оператором $L_{1}^{-1}$. Подействовав же на обе части уравнения (1) оператором $M_{0}^{-1}(I-Q)$, получим уравнение (4). 
3. Определение $\varepsilon$-управляемости. Уравнение (3) имеет тот же вид, что и (2), его $\varepsilon$-управляемость исследована в [7]. Целью данной работы является исследование $\varepsilon$-управляемости уравнения (4) и системы (3), (4), т.е. уравнения (1).

Решение системы согласно теореме 2 имеет вид

$$
x(t)=U^{t} x_{0}+\int_{0}^{t} U^{t-s} L_{1}^{-1} b^{1} u(s) d s-\sum_{k=0}^{p} H^{k} M_{0}^{-1} b^{0} u^{(k)}(t),
$$

где $p$ - порядок полюса (либо нуль в случае устранимой особой точки). Поэтому будем использовать управление $u(t) \in C^{p+1}([0, T] ; \mathbb{R})$ с соответствующим $p$.

Заметим, что в выражении (5) сумма первых двух слагаемьх является решением уравнения (3), а третье слагаемое - решение уравнения (4). Этот факт активно используется в дальнейшем.

Говоря об $\varepsilon$-управляемости системы, описьваемой некоторым уравнением, через $x(T)=x\left(T ; x_{0} ; u(t)\right)$ будем обозначать значение в момент времени $T$ решения задачи Коши с начальньм значением $x_{0}$ для соответствующего уравнения.

ОПРЕДЕЛЕНИЕ 2. Система назьвается $\varepsilon$-управляемой в нуль за время $T$, если для любых $x_{0} \in \mathscr{X}, \varepsilon>0$ существует функция $u(t) \in C^{p+1}([0, T] ; \mathbb{R})$, для которой $\left\|x\left(T ; x_{0} ; u(t)\right)\right\| \leqslant \varepsilon$.

ОПРЕДЕЛЕНИЕ 3. Система называется $\varepsilon$-управляемой из нуля за время $T$, если для любых $\widetilde{x} \in \mathscr{X}$ и $\varepsilon>0$ существует такая функция $u(t) \in C^{p+1}([0, T] ; \mathbb{R})$, что $\|x(T ; 0 ; u(t))-\widetilde{x}\| \leqslant \varepsilon$.

ОПРЕДЕЛЕНИЕ 4. Система назьвается $\varepsilon$-управляемой из любой точки в любую за время $T$, если для любых точек $x_{0} \in \mathscr{X}, \widetilde{x} \in \mathscr{X}$ и для любого $\varepsilon>0$ существует такая функция $u(t) \in C^{p+1}([0, T] ; \mathbb{R})$, что $\left\|x\left(T ; x_{0} ; u(t)\right)-\widetilde{x}\right\| \leqslant \varepsilon$.

Лемма 1. Для уравнения (3) понятия в-управляемости в нуль, из нуля и из любой точки в любую являются әквивалентными.

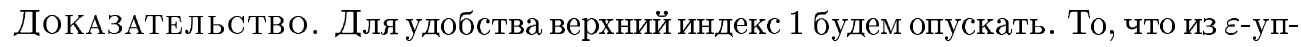
равляемости из любой точки в любую следуют $\varepsilon$-управляемость в нуль и $\varepsilon$-управляемость из нуля, очевидно.

Пусть система $\varepsilon$-управляема в нуль за время $T$, тогда

$$
\begin{gathered}
\forall x_{0} \in \mathscr{X}^{1} \quad \forall \varepsilon>0 \quad \exists u(t) \in C^{p+1}([0, T] ; \mathbb{R}): \\
\left\|x\left(T ; x_{0} ; u(t)\right)\right\|=\left\|U^{T} x_{0}+\int_{0}^{T} U^{T-s} L_{1}^{-1} b^{1} u(s) d s\right\| \leqslant \varepsilon .
\end{gathered}
$$

Для произвольного $\widetilde{x} \in \mathscr{X}^{1}$ возьмем вектор $x_{0}=-U^{-T} \widetilde{x} \in \mathscr{X}^{1}$ и получим

$$
\|x(T ; 0 ; u(t))-\widetilde{x}\|=\left\|\int_{0}^{T} U^{T-s} L_{1}^{-1} b^{1} u(s) d s+U^{T} x_{0}\right\| \leqslant \varepsilon .
$$

Следовательно, система $\varepsilon$-управляема из нуля за время $T$. 
Обратно, для $\varepsilon$-управляемой из нуля за время $T$ системы по произвольному $x_{0} \in \mathscr{X}^{1}$ выберем $\widetilde{x}=-U^{T} x_{0}$ и получим

$$
\varepsilon \geqslant\|x(T ; 0 ; u(t))-\widetilde{x}\|=\left\|x\left(T ; x_{0} ; u(t)\right)\right\| \text {. }
$$

Таким образом, система управляема в нуль.

Осталось показать, что из $\varepsilon$-управляемости в нуль за время $T_{1}$ и $\varepsilon$-управляемости из нуля за время $T_{2}$ следует $\varepsilon$-управляемость из любой точки в любую за время $T_{1}+T_{2}$. Действительно, из любой точки $x_{0}$ мы за некоторое время $T_{1}$ можем попасть в нуль:

$$
\left\|x\left(T_{1}\right)\right\|=\left\|U^{T_{1}} x_{0}+\int_{0}^{T_{1}} U^{T_{1}-s} L_{1}^{-1} b^{1} u(s) d s\right\| \leqslant \varepsilon \frac{e^{-T_{2}\left\|S_{1}\right\|}}{2\|P\|} .
$$

Затем, из нуля за некоторое время $T_{2}$ мы попадем в наперед взятую точку $\widetilde{x}$ с помощью функции управления $v(t)$ :

$$
\left\|\int_{0}^{T_{2}} U^{T_{2}-t} L_{1}^{-1} b^{1} v(t) d t-\widetilde{x}\right\| \leqslant \frac{\varepsilon}{2} .
$$

Здесь $U^{T_{2}}=e^{T_{2} S_{1}} P, S_{1}=L_{1}^{-1} M_{1}$, поэтому $\left\|U^{T_{2}}\right\| \leqslant\|P\| e^{T_{2}}\left\|S_{1}\right\|$. Пусть $t+T_{1}=s-$ общее время движения, после выхода из точки $x_{0}, t-$ время движения после выхода из точки нуль, тогда, считая $u\left(T_{1}+t\right)=v(t), t \in\left[0, T_{2}\right]$, получим

$$
\begin{aligned}
& \left\|x\left(T_{1}+T_{2}\right)-\widetilde{x}\right\|=\left\|U^{T_{1}+T_{2}} x_{0}+\int_{0}^{T_{1}+T_{2}} U^{T_{1}+T_{2}-s} L_{1}^{-1} b^{1} u(s) d s-\widetilde{x}\right\| \\
& \quad=\left\|U^{T_{2}}\left(U^{T_{1}} x_{0}+\int_{0}^{T_{1}} U^{T_{1}-s} L_{1}^{-1} b^{1} u(s) d s\right)+\int_{0}^{T_{2}} U^{T_{2}-t} L_{1}^{-1} b^{1} v(t) d t-\widetilde{x}\right\| \leqslant \varepsilon .
\end{aligned}
$$

ЗАмЕчАниЕ 1 . Ввиду леммы 1 в дальнейшем будем говорить просто об $\varepsilon$-управляемости за время $T$ системы (3), подразумевая под этим все три ее разновидности, рассмотренные нами.

Лемма 2. (i) Для системы (4) понятия в-управляемости из нуля за время Т и в-управляемости из любой точки в любую за время Т являются эквивалентными;

(ii) система (4) управляема в нуль за любое время $T$.

ДокАЗАТЕльСтво. Утверждение (i) очевидно, поскольку решение уравнения (4) не зависит от значения $x_{0}$.

В утверждении (ii) речь идет о точной $(\varepsilon=0)$ управляемости. Чтобы показать ее, достаточно взять $u(t) \equiv 0$.

ЗАмЕчАниЕ 2. Согласно утверждению (ii) леммы 2 понятие $\varepsilon$-управляемости в нуль для системы (4) является бессодержательным. Поэтому в дальнейшем под $\varepsilon$-управляемостью системы (4), а следовательно, и системы (1), будем подразумевать $\varepsilon$-управляемость из нуля или из любой точки в любую.

ПРЕДЛОЖЕНИЕ 1. Если система (1) в-управляема за время T, то $b^{0} \neq 0 u$ $b^{1} \neq 0$. 
ДокАЗАТЕЛЬСтво. Действительно, в противном случае одно из уравнений (3) или (4) является однородньц, а значит, не является $\varepsilon$-управляемым.

4. Одномерная $\varepsilon$-управляемость уравнения соболевского типа. Сначала приведем известные ранее факты о системе (3).

ТеОрема 3 [7]. Система (3) в-управляема за время $T$ в том и только том случае, когда линейная оболочка векторов

$$
\left\{L_{1}^{-1} b^{1}, S_{1} L_{1}^{-1} b^{1}, \ldots, S_{1}^{m} L_{1}^{-1} b^{1}, \ldots\right\}
$$

плотна в пространстве $\mathscr{X}^{1}$.

ДокАЗАтЕльство. Утверждение сразу следует из теоремы 1 [7].

Последовательность векторов $\left\{e_{m}\right\}_{m=0}^{\infty} \subset \mathscr{X}$ назовем базисом в пространстве $\mathscr{X}$, если каждый элемент $x \in \mathscr{X}$ однозначно представим в виде

$$
x=\sum_{m=0}^{\infty} a_{m} e_{m}
$$

где $a_{m}$ - некоторые коэффициенты.

СледСТВИЕ 1 [7]. Система (3) в-управляема за время T, если векторь

$$
\left\{L_{1}^{-1} b^{1}, S_{1} L_{1}^{-1} b^{1}, \ldots, S_{1}^{m} L_{1}^{-1} b^{1}, \ldots\right\}
$$

образуют базис в пространстве $\mathscr{X}^{1}$.

ЗАмечАниЕ 3 . Точная $(\varepsilon=0)$ управляемость при этом, вообще говоря, не имеет места согласно теореме 2 [8].

Перейдем к рассмотрению системы (4).

Лемма 3. Пусть оператор $M(L, \sigma)$-ограничен, а бесконечность - несущественная особая точка порядка р оператор-функиии $(\mu L-M)^{-1}$. Cистема (4) в-управляема за время $T$ тогда и только тогда, когда линейная оболочка векторов

$$
\left\{M_{0}^{-1} b^{0}, H M_{0}^{-1} b^{0}, \ldots, H^{p} M_{0}^{-1} b^{0}\right\}
$$

плотна в пространстве $\mathscr{X}^{0}$.

ДокАЗАТЕЛЬСтво. Прямое утверждение леммы сразу следует из вида решения

$$
x(t)=-\sum_{k=0}^{p} u^{(k)}(t) H^{k} M_{0}^{-1} b^{0} .
$$

Обратно, плотность линейной оболочки векторов

$$
\left\{M_{0}^{-1} b^{0}, H M_{0}^{-1} b^{0}, \ldots, H^{p} M_{0}^{-1} b^{0}\right\}
$$

означает, что для любого $\widetilde{x}^{0}$ и сколь угодно малого $\varepsilon>0$ существуют константы $c_{k}$, $k=\overline{0, p}$, такие, что

$$
\left\|\sum_{k=0}^{p} c_{k} H^{k} M_{0}^{-1} b^{0}-\widetilde{x}^{0}\right\| \leqslant \varepsilon .
$$

По ним построим искомую функцию управления

$$
u(t)=-\sum_{k=0}^{p} \frac{c_{k}}{k !}(t-T)^{k} .
$$


ЗАМЕЧАНИЕ 4. Из доказательства леммы видно, что из плотности линейной оболочки векторов $\left\{M_{0}^{-1} b^{0}, H M_{0}^{-1} b^{0}, \ldots, H^{p} M_{0}^{-1} b^{0}\right\}$ следует $\varepsilon$-управляемость системы (4) за любое время $T$.

TеОрема 4. Пусть оператор $M(L, \sigma)$-ограничен, а бесконечность - несущественная особая точка порядка р оператор-функиии $(\mu L-M)^{-1}$. Cистема (4) в-управляема тогда и только тогда, когда при некотором $q, 0 \leqslant q \leqslant p$, пространство $\mathscr{X}^{0}(q+1)$-мерно, а система векторов $\left\{M_{0}^{-1} b^{0}, H M_{0}^{-1} b^{0}, \ldots, H^{q} M_{0}^{-1} b^{0}\right\}$ является в нем базисом.

ДокАЗАТЕльСтво. Пусть $q$ - наименьшее число такое, что выполняется

$$
H^{q} M_{0}^{-1} b^{0} \neq 0, \quad H^{q+1} M_{0}^{-1} b^{0}=0 .
$$

Обозначим $H^{k} M_{0}^{-1} b^{0}=z_{k}, k=\overline{0, p}$. Так как $z_{k+m}=H^{m} z_{k}$, то $z_{k} \neq 0, k=\overline{0, q}, z_{l}=0$, $l=\overline{q+1, p}$. Пусть для некоторых $c_{k}, k=\overline{0, q}$,

$$
\sum_{k=0}^{q} c_{k} z_{k}=0
$$

Подействуем на это равенство оператором $H^{q}$ и получим $c_{0} z_{q}=0$. Отсюда $c_{0}=0$,

$$
\sum_{k=1}^{q} c_{k} z_{k}=0
$$

Подействовав оператором $H^{q-1}$, получим $c_{1}=0$ и т. д. Таким образом, векторы $z_{k}$, $k=\overline{0, q}$, линейно независимы.

Обозначим соответствующее $\varepsilon=1 / n$ управление $u(t)=u_{n}(t)$, его значения $u_{n}^{(k)}(T)=\alpha_{k}^{n}, k=\overline{0, q}, n \in \mathbb{N}$. В новых обозначениях получаем

$$
\forall \widetilde{x}^{0} \in \mathscr{X}^{0} \quad \forall n \in \mathbb{N} \quad\left\|\sum_{k=0}^{q} \alpha_{k}^{n} z_{k}-\widetilde{x}^{0}\right\|<\frac{1}{n} .
$$

Поэтому,

$$
\lim _{n \rightarrow \infty} \sum_{k=0}^{q} \alpha_{k}^{n} z_{k}=\widetilde{x}^{0} \quad \text { и } \quad \mathscr{X}^{0}=\overline{\operatorname{span}}\left\{z_{0}, z_{1}, \ldots, z_{q}\right\} .
$$

Обратно, согласно предположению для любого $\widetilde{x}^{0}$ существуют константы $c_{k}, k=\overline{0, q}$, такие, что

$$
\sum_{k=0}^{q} c_{k} H^{k} M_{0}^{-1} b^{0}=\widetilde{x}^{0}
$$

Построив по ним управление

$$
u(t)=-\sum_{k=0}^{q} \frac{c_{k}}{k !}(t-T)^{k}
$$

получим даже точную управляемость системы. 
СлЕДСтвИЕ 2. Пусть оператор $M(L, \sigma)$-ограничен, а бесконечность - несущественная особая точка порядка р оператор-функиии $(\mu L-M)^{-1}$. Тогда в-управляемость системы (4) равносильна ее точной управляемости.

ДокАЗАТЕЛЬСТвО. Из точной управляемости с очевидностью следует $\varepsilon$-управляемость.

Согласно предыдущей теореме из $\varepsilon$-управляемости системы следует, что

$$
\left\{M_{0}^{-1} b^{0}, H M_{0}^{-1} b^{0}, \ldots, H^{q} M_{0}^{-1} b^{0}\right\}
$$

- базис в пространстве $\mathscr{X}^{0}$. Используя доказательство теоремы 4 , получим точную управляемость.

Теперь можно сформулировать основной результат об одномерной $\varepsilon$-управляемости уравнения (1).

ТЕОрема 5. Пусть оператор $M(L, \sigma)$-ограничен, а бесконечность - несущественная особая точка порядка р оператор-функиии $(\mu L-M)^{-1}$. Пусть, кроме того, система (1) в-управляема. Тогда при некотором $q, 0 \leqslant q \leqslant p$, система векторов

$$
\left\{M_{0}^{-1} b^{0}, H M_{0}^{-1} b^{0}, \ldots, H^{q} M_{0}^{-1} b^{0}\right\}
$$

является базисом в пространстве $\mathscr{X}^{0}$, а линейная оболочка векторов

$$
\left\{L_{1}^{-1} b^{1}, S_{1} L_{1}^{-1} b^{1}, \ldots, S_{1}^{m} L_{1}^{-1} b^{1}, \ldots\right\}
$$

плотна в пространстве $\mathscr{X}^{1}$.

ЗАмечАниЕ 5. Справедливость утверждения, обратного к утверждению теоремы 5 , остается под вопросом, поскольку системами (3) и (4) одновременно управляет одна и та же функция $u(t)$.

5. Двумерная управляемость уравнения соболевского типа. В силу замечания 5 в случае уравнений соболевского типа более естественным представляется рассматривать управляемость уравнения

$$
L \dot{x}(t)=M x(t)+b^{0} u_{0}(t)+b^{1} u_{1}(t), \quad 0 \leqslant t \leqslant T .
$$

Здесь векторы $b^{0} \in \mathscr{X}^{0}, b^{1} \in \mathscr{X}^{1}$. Очевидно, что уравнение (6) также сводится к системе уравнений $(3),(4)$ с функциями $u_{0}(t), u_{1}(t)$ вместо $u(t)$ соответственно.

Согласно соображениям, изложенным в п. 3 , для этой системы ограничимся одним определением $\varepsilon$-управляемости.

ОПРЕДЕЛЕНИЕ 5. Систему (6) назовем $\varepsilon$-управляемой за время $T$, если для любых точек $x_{0} \in \mathscr{X}, \widetilde{x} \in \mathscr{X}$ и для любого $\varepsilon>0$ существуют такие функции

$$
u_{0}(t) \in C^{p+1}([0, T] ; \mathbb{R}), \quad u_{1}(t) \in L_{1}([0, T] ; \mathbb{R}),
$$

что $\left\|x\left(T ; x_{0} ; u_{1}(t), u_{0}(t)\right)-\widetilde{x}\right\| \leqslant \varepsilon$.

Здесь

$$
x\left(T ; x_{0} ; u_{1}(t), u_{0}(t)\right)=U^{T} x_{0}+\int_{0}^{T} U^{T-s} L_{1}^{-1} b^{1} u_{1}(s) d s-\sum_{k=0}^{p} H^{k} M_{0}^{-1} b^{0} u_{0}^{(k)}(T)
$$

- решение задачи Коши $x(0)=x_{0}$ для уравнения (6). 
Теорема 6. Пусть оператор $M(L, \sigma)$-ограничен, а бесконечность - несущественная особая точка порядка р оператор-функиии $(\mu L-M)^{-1}$. Cистема (6) в-управляема тогда и только тогда, когда при некотором $q, 0 \leqslant q \leqslant p$, система векторов

$$
\left\{M_{0}^{-1} b^{0}, H M_{0}^{-1} b^{0}, \ldots, H^{q} M_{0}^{-1} b^{0}\right\}
$$

является базисом в пространстве $\mathscr{X}^{0}$, а линейная оболочка векторов

$$
\left\{L_{1}^{-1} b^{1}, S_{1} L_{1}^{-1} b^{1}, \ldots, S_{1}^{m} L_{1}^{-1} b^{1}, \ldots\right\}
$$

плотна в пространстве $\mathscr{X}^{1}$.

СлЕДСТВИЕ 3. Пусть оператор $M(L, \sigma)$-ограничен, а бесконечность - несущественная особая точка порядка р оператор-функиии $(\mu L-M)^{-1}$. Если при некотором $q, 0 \leqslant q \leqslant p$, система векторов

$$
\left\{M_{0}^{-1} b^{0}, H M_{0}^{-1} b^{0}, \ldots, H^{q} M_{0}^{-1} b^{0}, L_{1}^{-1} b^{1}, S_{1} L_{1}^{-1} b^{1}, \ldots, S_{1}^{m} L_{1}^{-1} b^{1}, \ldots\right\}
$$

образует базис в пространстве $\mathscr{X}$, то система (6) в-управляема.

6. Пример. Пусть $\Omega \subset \mathbb{R}^{n}$ - ограниченная область с границей $\partial \Omega$ класса $C^{\infty}$. В цилиндре $\Omega \times \mathbb{R}$ рассмотрим задачу Коши-Дирихле

$$
\begin{aligned}
v(x, 0) & =v_{0}(x), & x & \in \Omega, \\
v(x, t) & =0, & (x, t) & \in \partial \Omega \times \mathbb{R}
\end{aligned}
$$

для уравнения Баренблатта-Желтова-Кочиной [9]

$$
(\lambda-\Delta) v_{t}(x, t)=\alpha \Delta v(x, t)+b(x) u(t), \quad(x, t) \in \Omega \times \mathbb{R},
$$

$\lambda \in \mathbb{R}, \alpha \in \mathbb{R}_{+}$, моделирующего фильтрацию жидкости в трещиновато-пористой среде. $\Phi$ ункция $u(t)$ скалярная.

Положим

$$
\mathscr{X}=\left\{v \in W_{p}^{k+2}: v(x)=0, x \in \partial \Omega\right\}, \quad \mathscr{Y}=W_{p}^{k},
$$

либо

$$
\mathscr{X}=\left\{v \in C_{p}^{k+2+\nu}: v(x)=0, x \in \partial \Omega\right\}, \quad \mathscr{Y}=C^{k+\nu},
$$

где $W_{p}^{l}=W_{p}^{l}(\Omega)$-пространство Соболева, $1<p<\infty, C^{l+\nu}=C^{l+\nu}(\Omega)-$ пространство Гёльдера, $0<\nu<1, l=0,1, \ldots$ В качестве операторов $L$ и $M$ возьмем $\lambda-\Delta$ и $\alpha \Delta$ соответственно. Функция $b(x) \in W_{p}^{k}$ или $b(x) \in C^{k+\nu}$.

В [1] показано, что при любых $\lambda \in \mathbb{R}, \alpha \in \mathbb{R}_{+}$оператор $M(L, \sigma)$-ограничен, причем $\infty$ - устранимая особая точка $L$-резольвенты $(\mu L-M)^{-1}$ оператора $M$.

Пусть $\left\{\varphi_{k}: k=1,2, \ldots\right\}$ - множество собственных векторов задачи Дирихле для оператора Лапласа $\Delta$, занумерованное по невозрастанию собственньг значений $\left\{\lambda_{k}\right.$ : $k=1,2, \ldots\}$ с учетом их кратности, а $\langle\cdot, \cdot\rangle$ - скалярное произведение в $L_{2}(\Omega)$.

В случае $\lambda \notin \sigma(\Delta)$ оператор $L$ непрерывно обратим и рассматриваемьй случай тривиален, поскольку уравнение приводится к виду (2). Поэтому, в дальнейшем будем считать, что $\lambda \in \sigma(\Delta)$. 
Для рассматриваемой системы имеют место равенства

$$
\begin{aligned}
\mathscr{X}^{0} & =\operatorname{span}\left\{\varphi_{k}: \lambda_{k}=\lambda\right\}, & \mathscr{X}^{1} & =\overline{\operatorname{span}}\left\{\varphi_{k}: \lambda_{k} \neq \lambda\right\}, \\
M_{0}^{-1} b^{0} & =\frac{1}{\alpha \lambda} \sum_{\lambda_{k}=\lambda}\left\langle b, \varphi_{k}\right\rangle \varphi_{k}, & S_{1}^{m} L_{1}^{-1} b^{1} & =\alpha^{m} \sum_{\lambda_{k} \neq \lambda} \frac{\lambda_{k}^{m}\left\langle b, \varphi_{k}\right\rangle \varphi_{k}}{\left(\lambda-\lambda_{k}\right)^{m+1}},
\end{aligned}
$$

где $m=0,1, \ldots$ Системы $(3)$ и $(4)$ в данной ситуации сводятся к системам

$$
\begin{gathered}
\left\langle v(\cdot, 0), \varphi_{k}(\cdot)\right\rangle=\left\langle v_{0}(\cdot), \varphi_{k}(\cdot)\right\rangle, \\
\left\langle v_{t}(\cdot, t), \varphi_{k}(\cdot)\right\rangle=\frac{\alpha \lambda_{k}\left\langle v(\cdot, t), \varphi_{k}(\cdot)\right\rangle}{\lambda-\lambda_{k}}+u(t) \frac{\left\langle b(\cdot), \varphi_{k}(\cdot)\right\rangle}{\lambda-\lambda_{k}}, \quad t \in \mathbb{R}, \\
k \in \mathbb{N}: \lambda_{k} \neq \lambda, \\
\left\langle v(\cdot, 0), \varphi_{k}(\cdot)\right\rangle=\left\langle v_{0}(\cdot), \varphi_{k}(\cdot)\right\rangle, \\
0=\alpha \lambda\left\langle v(\cdot, t), \varphi_{k}(\cdot)\right\rangle+u(t)\left\langle b(\cdot), \varphi_{k}(\cdot)\right\rangle, \quad t \in \mathbb{R}, \\
k \in \mathbb{N}: \lambda_{k}=\lambda,
\end{gathered}
$$

соответственно.

ЛЕмма 4. Система (11) управляема тогда и только тогда, когда $\lambda$ является собственным значением оператора Лапласа кратности 1 , а функция $b(x)$ не ортогональна соответствующей собственной функиии в смысле $L_{2}(\Omega)$.

ДокАЗАТЕЛЬСТво. Поскольку в данном случае бесконечность - устранимая особая точка оператор-функции $(\mu L-M)^{-1}$, то порядок особенности в бесконечности равен 1 и по теореме 4 пространство $\mathscr{X}^{0}$ должно быть одномерно. Это не так, если кратность $\lambda$ больше единицы. Осталось сослаться на предложение 1.

Собственные функции оператора Лапласа, образующие базис в $\mathscr{X}^{1}$, переобозначим как $\left\{\psi_{0}, \psi_{1}, \psi_{2}, \ldots\right\}$, соответствующие им $\lambda_{k}$ также перенумеруем. Обозначим также для $w \in \mathscr{X}^{1} w_{k}=\left\langle w, \psi_{k}\right\rangle, b_{k}=\left\langle b, \psi_{k}\right\rangle, k=0,1, \ldots$

Лемма 5. Если оператор $\Lambda: l^{2} \rightarrow l^{2}$, имеющий вид

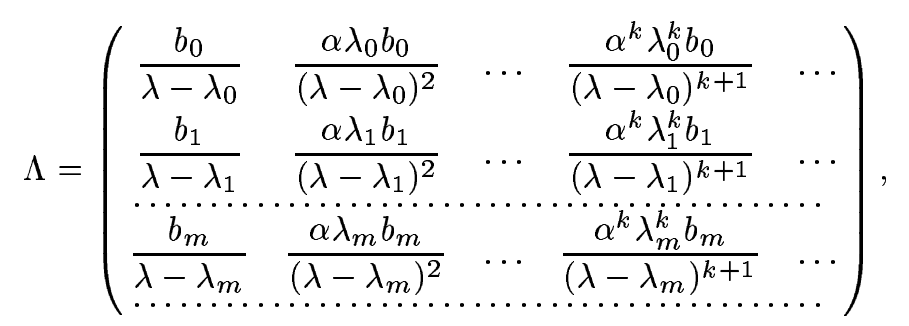

сюрвективен на своей области определения, то система (10) в-управляема. 
ДокАЗАтЕльСтво. Действительно, тогда для любой функции $w \in \mathscr{X}^{1}$ найдется последовательность чисел $\left\{c_{0}, c_{1}, c_{2}, \ldots\right\}$ такая, что

что означает равенство

$$
w_{k}=b_{k} \sum_{m=0}^{\infty} c_{m} \frac{\alpha^{m} \lambda_{k}^{m}}{\left(\lambda-\lambda_{k}\right)^{m+1}},
$$

$$
w=\sum_{m=0}^{\infty} c_{m} S_{1}^{m} L_{1}^{-1} b^{1} .
$$

Из него согласно теореме 3 следует $\varepsilon$-управляемость системы (10).

ЗАмЕчАниЕ 6 . Очевидно, что достаточное условие $\varepsilon$-управляемости системы (10), сформулированное в лемме 5 , очень жесткое. Оно исключает возможность существования кратных собственных значений и возможность ортогональности функции $b(x)$ хотя бы одной из функций $\psi_{k}$.

Рассмотрим уравнение

$$
\begin{aligned}
(\lambda-\Delta) v_{t}(x, t)= & \alpha \Delta v(x, t)+u_{0}(t) \sum_{\lambda_{k}=\lambda}\left\langle b(\cdot), \varphi_{k}(\cdot)\right\rangle \varphi_{k}(x) \\
& +u_{1}(t) \sum_{\lambda_{k} \neq \lambda}\left\langle b(\cdot), \varphi_{k}(\cdot)\right\rangle \varphi_{k}(x), \quad(x, t) \in \Omega \times \mathbb{R} .
\end{aligned}
$$

На основе лемм 4, 5 мы сможем дать достаточные условия для $\varepsilon$-управляемости системы (7), (8), (12), но не системы (7)-(9).

ТЕОремА 7. Пусть $\lambda$ является собственным значением оператора Лапласа кратности 1, функиия $b(x)$ не ортогональна соответствующей собственной функиии в смысле $L_{2}(\Omega)$, а оператор $\Lambda: l^{2} \rightarrow l^{2}$ сюрвективен на своей области определения. Тогда система (7), (8), (12) в-управляема.

\section{СПИСОК ЦИТИРОВАННОЙ ЛИТЕРАТУРЫ}

[1] Свиридюк Г. А. К общей теории полугрупп операторов // УМН. 1994. Т. 49. № 4. С. 47-74.

[2] Favini A., Yagi A. Degenerate differential equations in Banach spaces. New York: Marcel Dekker, Inc., 1999.

[3] Соболев С. Л. Об одной новой задаче математической физики // Изв. АН СССР. Сер. матем. 1954. Т. 18. С. 3-50.

[4] Демиденко Г. В., Успенский С. В. Уравнения и системы, не разрешенные относительно старшей производной. Новосибирск: Научная книга, 1998.

[5] Свиридюк Г. А. Задача Коши для линейного сингулярного операторного уравнения типа Соболева // Дифференц. уравнения. 1987. Т. 23. № 12. С. 2168-2171.

[6] Шолохович $\Phi$. А. Об управляемости линейных динамических систем // Изв. УрГУ. 1998. T. 10. № 1. С. 103-126.

[7] Куржанский А. Б. К управляемости в банаховых пространствах // Дифференц. уравнения. 1969. Т. 5. № 9. С. 1715-1718.

[8] Шолохович $\Phi$. А. Об управляемости в гильбертовом пространстве // Дифференц. уравнения. 1967. Т. 3. № 3. С. 479-484.

[9] Баренблатт Г. И., ЖКелтов Ю. П., Кочина И.Н. Об основных представлениях теории фильтрации в трещиноватых средах // ПММ. 1960. Т. 24. № 5. С. 58-73. 